\title{
A method of applying daily GCM outputs in assessing climate change impact on multiple day extreme precipitation for Brisbane River Catchment
}

\author{
$\underline{W . Y e}{ }^{a}$ and $Y \cdot L^{b}{ }^{b}$ \\ ${ }^{a}$ School of Science and Engineering, University of Waikato, New Zealand \\ Email:weive@waikato.ac.nz \\ ${ }^{b}$ ClimSystems Ltd. Hamilton, New Zealand
}

\begin{abstract}
Urban river floods are normally caused by consecutive days of extreme precipitation. It is expected that climate change will have significant impact on extreme precipitation because the warming atmosphere will alter the precipitation pattern by changing the global/regional hydrological cycle. The Intergovernmental Panel on Climate Change (IPCC) finds that there is a tendency for an increase in daily heavy precipitation events in many regions in the world, including some in which the mean precipitation is projected to decrease. Currently, the General Circulation Model (GCM) is still the most reliable tool for generating the future climate change scenarios, but GCM is still facing with the problems in simulating daily precipitation at regional or local scales, particularly in extreme precipitation events simulation, due to its coarse spatial resolution and the current incomplete understanding of the climate system. The poor performance in regional/local precipitation simulation makes it difficult of direct using GCM outputs in climate change impact on extreme precipitation change studies, because extreme precipitation event is most likely a localised phenomenon. Recently, research efforts have been put into the downscaling the GCM to support localised impact assessment. However, such downscaling models are either complex so computational demanding (dynamic downscaling) or require extensive of observed data (statistical downscaling). That has leads to a limited availability of model results which in most cases are not sufficient to fulfil a localised impact assessment needs.
\end{abstract}

Since the publication of IPCC AR4, many GCM daily simulation outputs have become publically available, which provide an opportunity to study the change impact on total precipitation amount at daily bases, either for one day or multiple days. Given that the direct application of GCM simulation data in assessing climate change impact on extreme precipitation is yet to be examined, the important question is then how to make use of the daily GCM results in order to obtain local daily precipitation statistics and their changes in the future. This is of major importance for the extreme precipitation properties since the upper tail of the precipitation distribution suffers most from the coarse resolution representation in the GCMs. Thus the focus of this study was given to examine the climate change impact on the extreme precipitation by linking the GCM daily simulation results with the local extreme precipitation observations based on a statistical approach. Due to the current incomplete understanding of the climate system, the GCM's precipitation generation mechanism is associated with high uncertainties. Pattern scaling has been proved to be an economic and efficient method to cope with such uncertainties in generating the range of future climate change scenarios from different GCMs. Based on this method, together with a GCM-ensemble probabilistic prediction, we developed a future extreme events generation method that is capable of addressing the range of uncertainties that caused by different GCM precipitation generation mechanisms. The method was applied to the study the climate change impact on the Brisbane River rainfall and flooding. Since the historical flooding in Brisbane River were caused by multipleday (typically $4-7$ consecutive day) extreme rainfall, the method was applied to analysis the 5-day maximum total rainfall for the 17 long term historical observation stations in the catchment. The results reveal a reinforced trend toward more intense extreme precipitation events into the future under climate change. The shortening of return periods for extreme precipitation events and greater intensity of such events has implications for planning and decision making of durable infrastructure along with emergency services planning, landuse regulation and building codes. This relates not only to possible flood mitigation strategies such as the potential need for additional flood mitigating infrastructure but also for the current built environment.

Keywords: Climate change, impact assessment, extreme precipitation event, flood. 


\section{INTRODUCTION}

Each year extreme rainfall events cause substantial flood damage, particularly in the highly urbanized regions. Climate change will have significant impact on extreme precipitation because the warming atmosphere will alter the precipitation pattern by change the global/regional hydrological cycle. To make an effective flood prevention and mitigation planning, it is critical to have the support information about how the future extreme precipitation event changes might be under the climate change impact. Currently, it is commonly agreed that General Circulation Model (GCM) is still the best tool in constructing climate change scenarios. However, the relatively coarse resolutions of GCMs may suitable for simulation of phenomena that manifest at synoptic scales, whereas extreme precipitation is of mesoscale rather than of synoptic scale. These have led to a great difficulty in applying the GCM simulation outputs directly in analysing the climate change impact on extreme precipitation event. Climate change is associated with the uncertainties caused by various emission scenarios and the differences among GCMs in precipitation simulations, particularly in extreme precipitation simulation. Such uncertainties are one of the challenges facing with climate change impact assessment but is crucial information that needed for any effective and efficient adaptation planning. A planning practice that based on single GCM output is generally considered insufficient, while scenarios from an ensemble of GCM results capable of illustrating the difference of future changes are recommended. Instead of using GCM outputs directly, since early 1990s (Santer et al. 1990) an alternative method has been developed in constructing future climate change scenarios. The method, known as pattern scaling, was originally envisaged as a temporary compromise to add a time component to an equilibrium experiment with a GCM, pending the availability of transient experiments, and also to permit the comparison of standardised spatial patterns from different GCMs. This technique has been proved to be useful for a comprehensive risk assessment of climate change when more and more GCM outputs have become publicly available (Mitchell 2003; Li et al. 2009). Pattern scaling offers the possibility of representing the whole range of uncertainties involved in future climate change projections based on various combinations of emission scenarios and GCM outputs, which allows cross model sensitivity analyses and uncertainty examinations to be conducted easily (TGICA 2007). Various versions of the technique have been widely used in mean temperature and precipitation change studies (Mitchell 2003; Rik Leemans, R. and Bas Eickhout, 2004; Ruosteenoja et al. 2007). The objective of this study is to extend the pattern scaling method to analysis the climate change impact on multiple day extreme precipitation events, which in most cases are the major source for urban flooding such as the 1974 and 2011 floods in Brisbane, Australia.

\section{METHOD}

\subsection{General Extreme Value (GEV) Distribution}

The intensity and frequency of extreme events are typically represented by their recurrence interval, or annual return intervals (ARI). These are values that are exceeded, on average, once every specified number of years. Extreme value statistical theory dictates that under very general assumptions regarding the parent distribution, the values in its tail must obey well defined distribution functions (Leadbetter et al., 1983). If extreme events are defined to be the maximum or minimum value encountered over a fixed period of time, for instance seasonally or annually, the GEV distribution function appropriately describes the distribution of such extreme values (Castillo, 1988).

Long term daily time series data is normally required to construct an annual maximum (minimum) data set that can then be fitted statistically with a pre-determined distribution function, i.e., the GEV distribution in this study. The GEV distribution is a three parameter function. A number of methods exist in estimating the GEV function parameters using distribution fitted to a sample of annual extremes. The method adopted in this study was Probability Weighted Moment (PWM) based estimators following Hosking et al. (1985). Having fitted the GEV distribution to a sample of annual extremes, the $T$-year return (ARI) value $X_{T}$ can be estimated from the quantile function (inverse of the cumulated distribution function).

\subsection{Pattern Scaling Method}

Pattern scaling method is based on the assumption that,

1. a simple climate model at global scale can accurately represent the global mean responses simulated by a GCM (Raper et al. 2001);

2. a wide range of climatic variables simulated by a GCM are in linear relationships with the global annual mean temperature change of the same GCM; and

3. such linear relationships are validate at different spatial and/or temporal scales (Mitchell 2003). 
The method has several advantages: e.g., the noise caused by internal variability is reduced and the information provided by GCM outputs performed with various forcing scenarios is utilized effectively. Clearly, in order to adequately assess the impact of climate change on extreme precipitation, the characteristics of GCM simulated precipitation and its relationship with global warming need to be evaluated. The magnitude of changes in precipitation extremes simulated by GCMs was found to have a linear relationship with the strength of GHG emissions or in proportion with the global warming trend for Australia and South America by Alexander and Arblaster (2009) and Tebaldi et al (2006) respectively, which is in general agreement with the linear response assumption of pattern scaling method.

For climate change impact assessment, results based on an ensemble model approach is preferred to a single GCM model outputs, as a single projection of future climate made with even the most sophisticated GCM can be of limited use for impact assessment. As pointed out by Palmer and Räisänen (2002) that ensemble-based probabilistic predictions, as used in short- and medium-term forecasts of weather and climate, were more useful than deterministic forecasts using a 'best guess' scenario to address this sort of problem. One example of the ensemble method application of this to extreme event analysis can be found from Kharin et al (2007).

\subsection{Extreme event scenario generation}

For each GCM grid $(i, j)$, the PWM was used to estimate the GEV function parameters for the GCM simulated baseline and future periods. For a target return period $T$, the difference of its future projected and the baseline, a.k.a observed extreme precipitation value $X_{T}$ is calculated as:

$$
\Delta X_{T(s y i j)}=X_{T(s y i j)}-X_{T(i j)}
$$

where $X_{T(i j)}$ is the $T$ ARI value of the GCM baseline for grid $(i, j)$, which was derived from applying GEV function to GCM's simulation period of 1960-2000; $X_{T(s y j)}$ is the projected $T$ value for future year $y$ under the emission scenario $s$ for the same grid. It was calculated from applying GEV function to 20 or 30 years of GCM simulated data (depend on data availability) that takes the future year $y$ as the central time point.

Since the increased radiative forcing drives the global warming trend, the pattern scaling method may be described as: for a given $X_{T}$, its anomaly $\Delta X_{T}^{*}$ for a particular grid $(i, j)$, and year $(y)$ under an emission forcing scenario $(s)$ can be derived as:

$$
\Delta X_{T(s y i j)}^{*}=\Delta T_{(s y)} \cdot \Delta X_{T(i j)}^{\prime}
$$

where $\Delta T_{(s v)}$ being the annual global mean temperature change between year $y$ and baseline, which is typically simulated by a simple global climate model such as MAGICC (Wigley and Raper, 2002); and $\Delta X_{T(i j)}^{\prime}$ is the change rate of $X_{T}$ at grid $(i, j)$ in response to annual global mean temperature change. According to pattern scaling assumption, for a given GCM, $\Delta X_{T(i j)}^{\prime}$ should be obtained from any one of its simulation runs. However, such a consistent linear response is rarely found for a given available GCM data. One possible reason would be that the current available simulation period of 20 year from any GCM may not be long enough for extracting $\Delta X_{T(i j)}$ with sufficient statistical significance. On the other hand, one could also argue that this may be because the change rate of $X_{T}$ is not liner to the annual global temperature change in nature. Indeed, further research is required to look into the relationships between the change rate of various climate variables and the global mean temperature changes. However, such a research requires extensive GCM experiments with purposely designed input and outputs requirements, which may not become reality in the near future. Nevertheless, pattern-scaling does not seem to be a very large source of error in constructing regional climate projections for extreme scenarios (Ruosteenoja et al, 2007). Assuming the validity of the pattern scaling method, for a given GCM it is possible to take all available GCM outputs in calculating $\Delta X_{T(i j)}$ to reduce the effects of the GCM internal variability from different sampled emission scenarios and time periods. In this study, a least squares regression as described by Mitchell (2003) and Ruosteenoja et al. (2007) was followed:

$\Delta X_{T(i j)}^{\prime}=\frac{\sum_{y=1}^{m} \sum_{s=1}^{n} \Delta T_{(s y)} \cdot \Delta X_{T(s v i j)}}{\sum_{y=1}^{m} \sum_{s=1}^{n}\left(\Delta T_{(s y)}\right)^{2}}$

where $n$ is the number of emission scenarios and $m$ is the number of future simulation periods from a GCM. In this study, for all GCMs used, results from three SRES scenarios (A1B, A2, and B1) and 2 sample periods 
(2046-2065 and 2081-2100) were available, i.e $n=3$ and $m=2$. For a given $T$ ARI value, a spatial $\Delta X_{T}^{\prime}$ was calculated by applying Equ. 3 to each GCM grid $(i, j)$.

After $\Delta X_{T(i j)}^{\prime}$ was obtained for a given grid $(i, j), \Delta X_{T(s y j)}^{*}$ can be calculated from Equ. 2 with a given $\Delta T_{(s y)}$ (the commonly used ones are IPCC SRES), and the future extreme event values for the grid can then be derived from:

$$
X_{T(s v i j)}=\widetilde{X}_{T(i j)}+\Delta X_{T(s v i j)}^{*}
$$

where $\widetilde{X}_{T(i j)}$ is the observed $T$ ARI value.

In order to construct the GEV function for a future year $y, \Delta X_{T(s y j)}$ was calculated for 7 ARIs, i.e., $T=(2,5,10$, $20,50,100)$ based on Eqa. 4. The GEV function parameters for the future year $y$ can then be obtained by fitting the 7 extreme values to GEV function using Levenberg-Marquardt algorithm (Press et al., 1997).

Apart from the differences in emission scenarios, another major source of uncertainty for future climate projection is the different projections from different GCMs. For the same region or a localised area represented by a GCM grid, the change pattern of extreme precipitation values can vary significantly among GCM simulations, representing a large inter-model uncertainty. As a result, large ensembles of GCM predictions sampling the widest possible modelling range are needed for comprehending the range of uncertainties. The quantified uncertainty range provides important information for identifying effective and efficient adaptation measures against potential negative climate change impact.

Unban flooding is typically caused by multiple day extreme precipitation. For example, the 1974 and 2011 Brisbane floods were caused by 4 and 7 consecutive day heavy rainfalls respectively. In this study, we analysed the climate change impact on 5 day rainfall for Brisbane River catchment. A nonparametric adjusted bootstrap (BCa) method (Davison and Hinkley 1997) was adopted to estimate the $95 \%$ confidence interval for the baseline GEV. The year 2100 was chosen for demonstration of the future extreme precipitation change. Given the high uncertainties due to the large variation of the GCM outputs in projecting the precipitation change for the region, the median value from ensemble of all the available GCM model outputs was used as a represent of the 'best guess' of future change scenario.

\section{DATA}

The Brisbane River catchment forms in the Brisbane and Cooyar Ranges of the Great Divide within the coordinate of $26^{\circ} 39^{\prime} \mathrm{S}$ to $27^{\circ} 24^{\prime} \mathrm{S}$ and $152^{\circ} 22^{\prime} \mathrm{E}$ to $153^{\circ} 09^{\prime} \mathrm{E}$, with an area about 13400 $\mathrm{km}^{2}$. The Brisbane City straddles the river near its mouth (Figure 1). The catchment comprises six sub-catchments: the Stanley, Lockyer, Bremer, Upper Brisbane, Middle Brisbane and metropolitan sub-catchments. A total of 17 precipitation stations which have long term daily precipitation observation were selected for this study. The observed daily station data was firstly aggregated for every 5 consecutive days to construct a 5 day total rainfall time series. A daily time series data was obtained from the averaged of the 17 stations and used to build the baseline GEV distribution function for the catchment. Table 1 lists the name and location for the stations. Each station has more than 60 years of observation, with Esk Post Office (40075) and Lowood Don Street (40120) have the longest of 124 years, and Mt Nebo Post Office (40147) the shortest of 64 years. The extreme precipitation event of January 2011 was added to the observation, assuming it will be

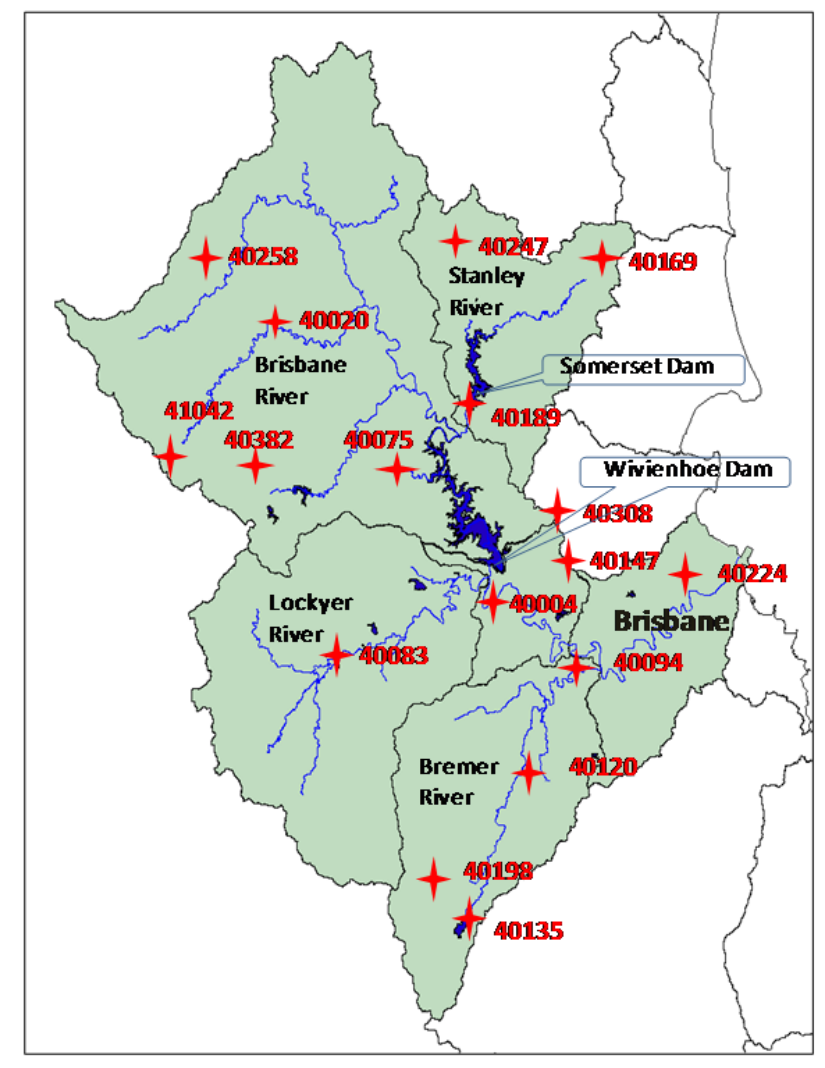

Figure 1: Brisbane River catchment and the location of the 17 rainfall stations (represented by Station ID) 
Ye and Li, Climate change impact on floods resulted from multi-day extreme precipitation event

Table 1: The 100 year return value of 5 day total extreme precipitation derived from GEV distribution and observed total precipitation that caused 1974 and 2011 floods. The top 8 columns are stations in the North.

\begin{tabular}{lcrrrrr}
\multicolumn{1}{c}{ Station Name } & ID & Latitude & Longitude & \multicolumn{2}{c}{ Extreme precipitation (mm) } \\
Yarraman Post Office & 40258 & -26.84 & 151.98 & 308 & 252 & 475 \\
Lindfield & 40247 & -26.84 & 152.58 & 566 & 330 & 576 \\
Peachester Woodford Rd & 40169 & -26.84 & 152.88 & 797 & 678 & 824 \\
Blackbutt Post Office & 40020 & -26.88 & 152.10 & 362 & 352 & 439 \\
Haden Post Office & 41042 & -27.22 & 151.88 & 258 & 208 & 269 \\
Crows Nest & 40382 & -27.27 & 152.06 & 354 & 276 & 450 \\
Esk Post Office & 40075 & -27.24 & 152.42 & 464 & 449 & 427 \\
Somerset Dam & 40189 & -27.12 & 152.56 & 447 & 305 & 361 \\
Mt Glorious Fahey Rd & 40308 & -27.33 & 152.77 & 995 & 1318 & 648 \\
Mt Nebo Post Office & 40147 & -27.40 & 152.79 & 852 & 1118 & 519 \\
Alderley & 40224 & -27.42 & 153.00 & 552 & 553 & 329 \\
Amberley AMO & 40004 & -27.63 & 152.71 & 413 & 481 & 282 \\
Gatton Allan Street & 40083 & -27.54 & 152.30 & 316 & 322 & 339 \\
Harrisville Post Office & 40094 & -27.81 & 152.67 & 327 & 446 & 179 \\
Lowood Don Street & 40120 & -27.46 & 152.57 & 420 & 391 & 550 \\
Tarome & 40198 & -27.98 & 152.51 & 462 & 472 & 250 \\
Moogerah Dam & 40135 & -28.03 & 152.55 & 473 & 437 & 285
\end{tabular}

the biggest event for the year. Also lists in Table 1 are the 100 ARI values of 5 day total extreme rainfall calculated from baseline GEV, as well as the total rainfall that caused 1974 and 2011 floods.

In order to examine the climate change impact on annual daily maximum precipitation event, a time series precipitation data in daily time step is required from GCM simulations. So far, there are 12 GCMs that have their daily simulation results available, which include the baseline period (1960-2000) and 2 future periods (2046-2065 and 2081-2100) under 3 SRES scenarios (A1B, A2 and B1). All the 12 GCM outputs were firstly re-formatted to a unified $0.5^{\circ} \times 0.5^{\circ}$ grid using a bi-linear interpolation from their original resolution. The change pattern $\Delta X_{T}^{\prime}$ obtained at this resolution showed a great deal of noise between neighbouring grids, therefore a $3 \times 3$ grid smoothing process was introduced to reduce such a noise level.

\section{RESULT}

Figure 2 shows both the baseline GEV distribution and the 2100 GEV distribution projected by the median change value of the ensemble of the 5-day extreme precipitation for the catchment. Also shown in Figure 2 is the $95 \%$ confidence levels for the baseline GEV distribution based on a 10000 bootstrap sampling of the observed values. Compared to the baseline GEV, climate change makes extreme events at all level to become more intensified or more frequent in the future. However, all the projected changes from the Low GCM projection (based on SRES B1 with low climate change sensitivity) is still inside the upper bound of the $95 \%$ confidence interval, whereas the projected changes based on High GCM projection (based on emission scenario SRES A1FI with high climate change sensitivity) is already beyond the upper bound of the interval. The enlarged departures of the lines between observed and GCM projected GEV functions towards the upper tail of the distribution indicated an even stronger climate change effects when the precipitation event becomes more extreme. The baseline intensity of the 100 ARI was $445 \mathrm{~mm}$ and changed to $508 \mathrm{~mm}$ and $584 \mathrm{~mm}$ under low and high GCM projection scenarios respectively, which represent a potential range of intensity increase between 14 to $31 \%$. The frequency of the 100 ARI of baseline changed to 57 years and 37 years under the low and high GCM projection scenarios respectively, which are significant frequency increases between 43 to $63 \%$. We also calculated GEV for each station individually and derived their 100 ARI in comparison with the observed extreme rainfall that caused 1974 and 2011 floods and listed the results in Table 1.

\section{DISCUSSION AND CONCLUSIONS}

Extreme precipitation is a localized and complex phenomenon. Given the size of Brisbane catchment, its floods might be actually caused by different rainfall regime. In 1974 flood, most stations in the south observed a rainfall larger than 100 ARI value but were not recorded by stations in the north. Similar situation was again observed for 2011 flood but in a total opposite situation (Table 1). Though different, these extreme precipitation 
regimes can all lead to severe urban flood. At the catchment level, this research shows that the extreme precipitation event will shift to become more intensified and frequent due to climate change, particularly with the high change scenarios. However, the GEV distribution derived from the observed data shows a large $95 \%$ confidence level at the tail, hence the changes for those most extreme events may likely still inside the upper $95 \%$ bound of current GEV prediction.

\section{ACKNOWLEDGMENTS}

The climate data is provided by Bureau of Metrology (BOM), Australia. CLIMsystem Ltd provides the SimCLIM software system, which was used in generating the climate change scenario and for the extreme event analysis.

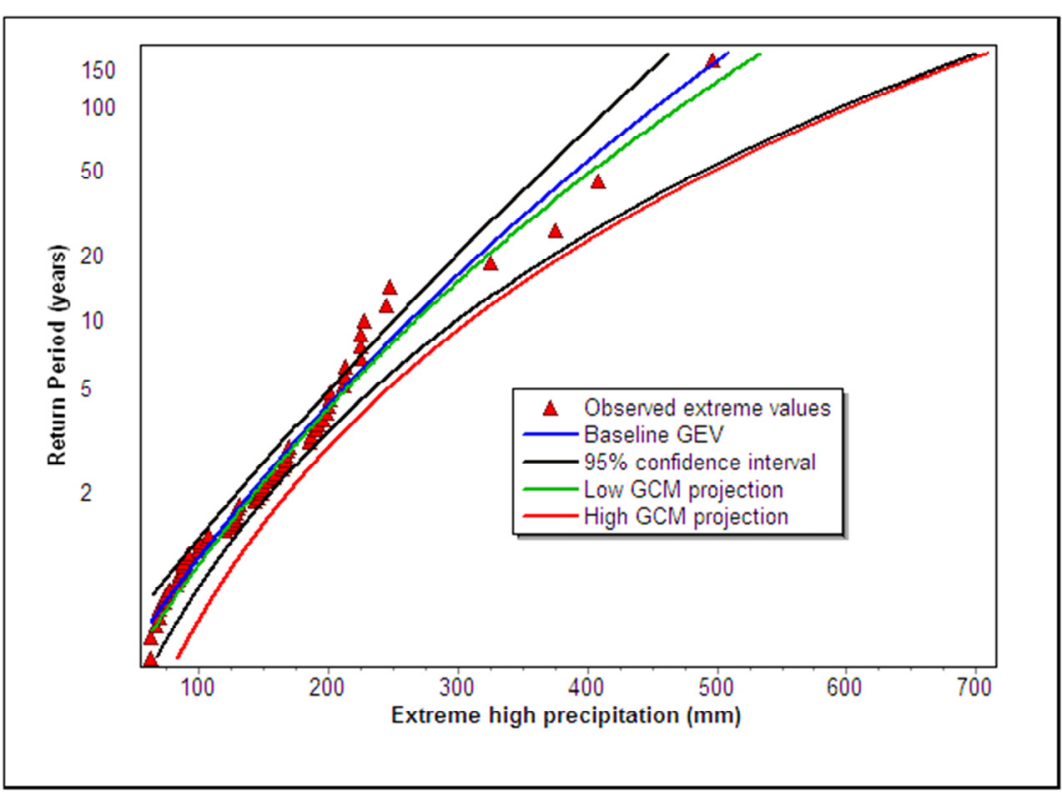

Figure 2: The baseline and year 2100 projection of GEV distributions of annual maximum of 5-day extreme high precipitation for Brisbane Rive Catchment. Low GCM projection was built on SRES B1 scenario with low climate change sensitivity; High GCM projection was built on SRES A1FI scenario with high climate sensitivity.

\section{REFERENCES}

Alexander, L.V. and Arblaster, J.M. (2009). Assessing trends in observed and modelled climate extremes over Australia in relation to future projections. Int. J. Climatol., 29, 417-435. DOI: 10.1002/joc.1730, 2009.

Davison, A. C. and D. V. Hinkley (1997) Bootstrap Methods and Their Applications. Cambridge University Press, $582 \mathrm{pp}$.

Castillo, E. (1988). Extreme value theory in engineering. Academic Press, Boston.

Kharin, V.V., Zwiers, F.W., Zhang, X., and Hegerl, G.C. (2007). Changes in temperature and precipitation extremes in the IPCC ensemble of global coupled model simulations. J. Clim., 20(8), 1419-1444. DOI: 10.1175/JCLI4066.1

Leadbetter, M.R., Lindgren, G. and Rootzen, H. (1983) Extremes and related properties of random sequences and processes. Springer-Verlag, New York, Inc.

Rik Leemans, R. and Bas Eickhout, B (2004) Another reason for concern: regional and global impacts on ecosystems for different levels of climate change Global Environmental Change 14: 219-228

Mitchell, T.D. (2003). Pattern scaling: An examination of the accuracy of the technique for describing future climates. Clim. Chang., 60, 217-242, 2003.

Palmer, T.N. and Räisänen, J. (2002) Quantifying the risk of extreme seasonal precipitation events in a changing climate. Nature 415, 512-514.

Press, W.H., Teukolsky, S.A., Vetterling, W.T. and Flannery, B.P. (1997) Numerical Recipes in C. Cambridge University Press.

Ruosteenoja, K., Tuomenvirta, H., Jylhä, K. (2007). GCM-based regional temperature and precipitation change estimates for Europe under four SRES scenarios applying a super- ensemble pattern-scaling method. Climat. Chang., 81, 193-208. DOI: 10.1007/s10584-006-9222-3.

Santer, B.D., Wigley, T.M.L., Schlesinger, M.E., and Mitchell, J.F.B. (1990). Developing climate scenarios from equilibrium GCM results, MPI Report Number 47, Hamburg.

Task Group on Data and Scenario Support for Impact and Climate Assessment (TGICA) (2007). Intergovernmental Panel on Climate Change: General guidelines on the use of scenario data for climate impact and adaptation assessment, Version 2

Tebaldi, C, Hayhoe, K., Arblaster, J.M. and Meehl, G.A. (2006). An intercomparison of model-simulated historical and future changes in extreme events. Climat. Chang., 79, 185-211.

Wigley T.M.L. and Raper S.C.B. (2002) Reasons for larger warming projections in the IPCC third assessment report. J Clim. 15:2945-2952 